NASA Technical Memorandum 103249

AIAA-90-0438

\title{
Euler Analysis Comparison With \\ LDV Data for an Advanced Counter- \\ Rotation Propfan at Cruise
}

Christopher J. Miller and Gary G. Podboy

Lewis Research Center

Cleveland, Ohio

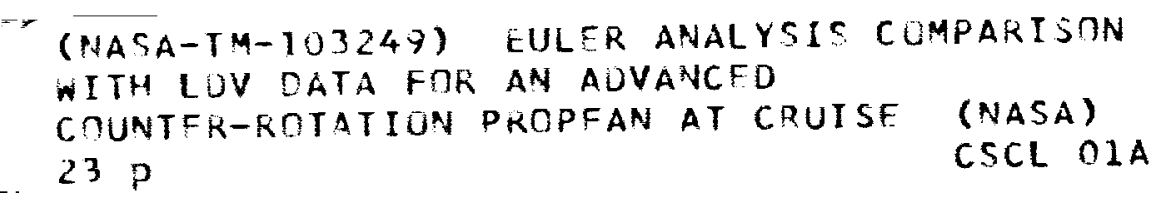

Prepared for the 8th Applied Aerodynamics Conference sponsored by the American Institute of Aeronautics and Astronautics Portland, Oregon, August 20-22, 1990 
作 $=$

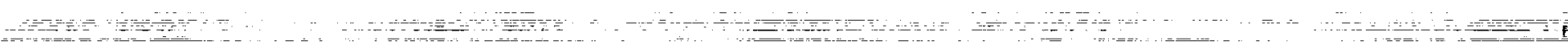

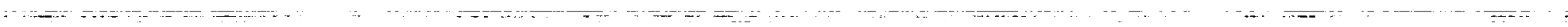

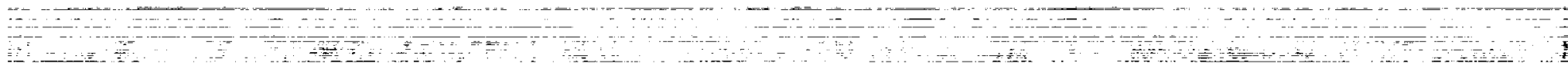
$\left[\begin{array}{ccc}\ldots & \ldots\end{array} \ldots\right.$

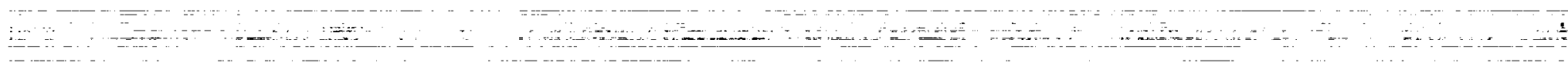

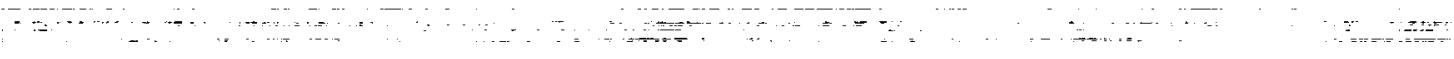

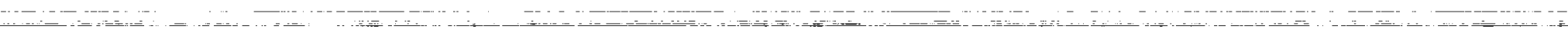




\title{
Euler Analysis Comparison With LDV Data For An Advanced Counter-Rotation Propfan At Cruise
}

\author{
Christopher J. Miller and Gary G. Podboy \\ National Aeronautics and Space Administration \\ Lewis Research Center \\ Cleveland, Ohio
}

\section{Summary}

A fine mesh Euler solution of the F4/A4 UDF model flowfield is compared with LDV data taken in the NASA Lewis 8- by 6-Foot Supersonic Wind Tunnel. The comparison is made primarily at one axial plane downstream of the front rotor where the LDV particle lag errors are reduced. The agreement between measured and predicted velocities in this axial plane is good. The results show that a dense mesh is needed in the centerbody stagnation region to minimize entropy generation that weakens the aft row passage shock. The predicted radial location of the tip vortex downstream of the front rotor agrees well with the experimental results but the strength is overpredicted. With 40 points per chord 1 ine, the integrated performance quantities are nearly converged, but more points are needed to resolve passage shocks and flow field details.

\section{Introduction}

Many computational codes [1-6] predict the aerodynamic and acoustic characteristics of advanced ducted and unducted propeller/fan geometries. The methodologies employed in these codes, however, require some simplifying assumptions regarding the nature of the flow through these machines. Over the years, advances in computational techniques and computer technology have permitted the use of fewer assumptions that, in turn, allow for a more realistic modeling of the complex three-dimensional flowfields.

The fully three-dimensional Euler solvers available today approximate the actual flow physics by neglecting viscous effects. Before these codes can be used extensively in the design process though, it is necessary to identify their limitations quantitatively. One way to do this is by making detailed comparisons between flowfield predictions and experimental data. The comparison here is between predictions from an Euler code and the LDV measured flowfield in the tip region of an advanced counterrotation pusher propfan. The operating point is near the high Mach number design point $(0.72)$ at cruise condition blade loadings. At this condition the blade tips are operating in a relative flow of Mach 1.08. Of interest are the strong tip vortices and the blade wakes from the front row that are interacting with the aft row. In the design process it is important to predict these flow features for acoustic predictions. 


\section{Symbols}

$A_{A} \quad$ propeller annulus area, $\mathrm{ft}^{2}$

$C_{p} \quad$ power coefficient; $P /\left(\rho_{0} \cdot n^{3} \cdot D^{5}\right)$

$C_{v}$ specific heat at constant volume

D propeller diameter, ft

IL maximum number of axial points

ILE leading edge axial index

ITE trailing edge axial index

$J$ advance ratio; $V_{0} /(n \cdot D)$

$\mathrm{JL}$ maximum number of radial points

JTIP tip radial index

$\mathrm{KL}$ maximum number of circumferential points

n rotational speed, $\mathrm{rev} / \mathrm{sec}$

$P \quad$ power, $\mathrm{ft}-1 \mathrm{~b} / \mathrm{sec}$

PQA power coefficient; $P /\left(\rho_{0} \cdot n^{3} \cdot D^{3} \cdot A_{A}\right)$

$p_{3} \quad$ static pressure, $1 \mathrm{~b} / \mathrm{ft}^{2}$

$\mathrm{p}_{\mathrm{t}} \quad$ total pressure, $1 \mathrm{~b} / \mathrm{ft}^{2}$

s entropy; $c_{v} \ln \left[\left(p / p_{\infty}\right) /\left(\rho / \rho_{\infty}\right)^{\gamma}\right]$

$T$ thrust, ib

TQA thrust coefficient; $T /\left(\rho_{0} \cdot n^{2} \cdot D^{2} \cdot A_{A}\right)$

$t_{s} \quad$ static temperature, degrees Rankine

$t_{t} \quad$ total temperature, degrees Rankine

$v$ velocity, $\mathrm{ft} / \mathrm{sec}$

$V_{0} \quad$ standard day corrected free-stream (axial) inflow velocity, $\mathrm{ft} / \mathrm{sec}$

B local blade angle, degrees

$\beta_{3 / 4}$ blade angle at 75 percent of tip radius, degrees

$\gamma \quad$ ratio of specific heats

$\rho \quad$ air density, slugs $/ \mathrm{ft}^{3}$

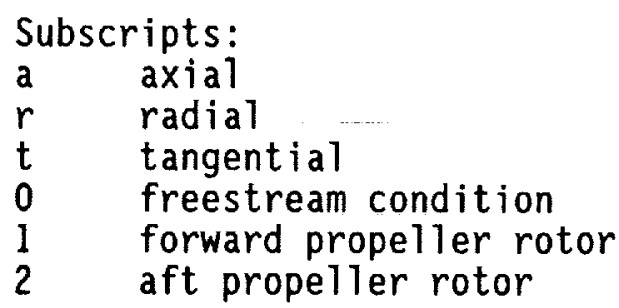

\section{Experimental Apparatus and Procedure}

\section{Test Facility}

The experimental data was taken in the NASA Lewis 8- by 6-Foot Supersonic Wind Tunnel. Reference 7 describes the tunnel in detail. A balance chamber encloses the 8-ft-high by 6-ft-wide test section. The walls, floor, and ceiling of the test section contain holes that provide a total porosity of approximately six percent. These holes are designed to minimize model-wall interactions at high subsonic speeds. For the LDV testing, the test section wall has a 26.5 inch diameter optical window that reduces the porosity and changes the free-stream Mach number slightly. While a recent tunnel

calibration measured this effect on Mach number, the analysis does not include the effect of the window. Instead the Mach number used was the tunnel Mach corrected for the presence of the porous walls. 


\section{Test Model}

The model used in this test is of a counterrotating pusher propeller. Except for the actual blade type and rotor-to-rotor spacing, this model is similar to the full-scale Unducted Fan (UDF) developed by General Electric. A detailed discussion of the propeller test rig and UDF model is in reference 8. The model had eight blades of the F4 design mounted in the front rotor and eight A4 blades installed in the aft. Table I 1ists the specifications for the $\mathrm{F} 4 / \mathrm{A} 4$ configuration design point.

The blade setting angles and the shaft speeds are constant throughout the LDV test program. During this testing, the model and tunnel operating conditions were read and recorded 83 times. Because of an apparent transducer malfunction, the power absorbed by the front row started to drift after the first 20 readings. The Euler predictions were generated to simulate the condition represented by the average of the overall performance characteristics for the first 20 readings. These values are 1 isted in table II.

\section{Laser Velocimeter}

A four-beam, two-color, on-axis backscatter LDV system was used to obtain the experimental data which was presented in reference 9 and will be used in these comparisons. This LDV system measured, at most, two components of velocity at one time. To measure all three components - axial, radial and tangential - data had to be obtained at the desired measurement locations in each of two different planes. These measurement planes are illustrated in figure 1. The time-averaged flow field was assumed to be axisymmetric, allowing the different velocity components to be obtained independently at different circumferential locations about the model centerline. Axial and tangential velocities were computed from measurements made with the probe volume positioned in the horizontal plane along the centerline of the model. Radial velocities were measured above the model in the vertical plane passing through the model centerline. Also illustrated in figure 1 is the sign convention used: axial velocities in the downstream direction are positive; tangential velocities in the direction of rotation of the front rotor are negative; and radial velocities outward away from the nacelle are positive.

The cross-marks in figure 2 show the axial and radial locations at which the LDV data were acquired. Data were obtained in nine constant axial planes outside of the blade rows and at three constant radial stations within each blade row. As explained in reference 9, all three velocity components were not acquired at all of the measurement locations. For each component which was measured, 2000 velocity measurements were obtained per location. An angle encoder was used to tag each velocity measurement with the angular position of either one of the two rotors. This encoder divided the 360 degrees of a complete rotor revolution into 4096 bins. During data acquisition each velocity measurement was sorted into one of these bins. As part of the data reduction process, the 2000 velocities were ensemble averaged into 256 bins ( 32 bins per blade pitch). The data of the eight blade passages was then phase lock averaged into one composite blade passage of 32 bins. A

circumferentially averaged velocity was also determined by computing the mean 
of the 32 phase lock averaged velocities. For more details on the data acquisition and data reduction procedures see reference 9 . These phase lock averaged and circumferentially averaged velocities will be compared to Euler solutions.

It should be pointed out that a correction has been applied to the radial velocities presented herein that was not applied to the data of reference 9. This correction is for a bias error that may be related to the use of a Bragg cell in the LDV transmitting optics. A comparison of tangential velocities measured with and without the Bragg cell (not shown here), illustrated the problem. During the test, tangential velocities were measured with the LDV system configured as both a one- and two-component system. In the two component configuration, tangential velocities are resolved from velocity components measured at $+/-45$ degrees from the horizontal. As a one-component system, the vertical velocity component (either tangential or radial depending on location) is measured directly. In this case a Bragg cell in the optical path sets the fringes within the probe volume in motion at a velocity proportional to the $40 \mathrm{MHz}$ cell frequency. Measurements taken without the Bragg cell showed the expected result of no swirl existing in the flow upstream of the propeller. Tangential velocities acquired with the Bragg cell, however, showed a negative swirl (opposite the direction of rotation) far upstream of the front rotor. In fact, the velocities measured with the Bragg cell consistently differed by 15 to 19 feet/second from those measured without the Bragg cell for all measurement locations. Whether or not the Bragg cell itself is the cause of these differences is not known; however, since this data is not consistent with what was expected to occur upstream of the rotor, it is thought to be in error. While the tangential velocities acquired with the Bragg cell presented in reference 9 were corrected for this bias, the radial velocities were not. The radial velocities presented in this report have been corrected by reducing each by $19 \mathrm{ft} / \mathrm{sec}$.

\section{Numerical Procedure}

The three-dimensional Euler code developed by Adamczyk, et al, [1,2] was used to generate the numerical predictions described here. This code solves for the three-dimensional flow through an "average" passage of a blade row. The algorithm uses a modified Jameson finite volume scheme with a four stage Runge-Kutta integration to solve for the flow through a single blade row. Distributed body forces and energy terms are applied to the cells swept out by the other blade rows to represent those rows during a calculation. Once the solution for the given row converges, the body forces and energy sources for the current row are updated from the axisymmetric average of the 3-D solution. The measure of convergence in the global sense is the mean squared difference $\left(1_{2}\right.$-norm) between two flow solutions.

The outer loop over the blade rows continues until $1_{2}$-norms for the 3-D flows about each row fall below $10^{-3}$ of the original values. Previous comparisons of experimentally measured nacelle surface pressures with those calculated using this code for a counterrotating propeller operating at high speed have shown good agreement [10]. 


\section{Results}

\section{Grid Study}

A grid study helped to determine the minimum number of flowfield points needed for a converged solution. The dependent variables for this study were the integrated thrust and power coefficients for each row. The independent variable was the number of points $N$ along a chord line. As $N$ changes, the number of mesh points in all directions are scaled to maintain cell aspect ratio. Figure 3 presents the dependence of $C_{p}$ on mesh density $(1 / N)$. The first three meshes with 10, 20, and 40 points (meshes 1,2, and 4) are coarse near the centerbody stagnation point. Also, the grid points are clustered near the leading and trailing edges, with no clustering at the passage shock locations. The mesh dimensions for these three meshes, as well as the meshes used later, are listed in more detail in Table III.

The 3-D flowfields also show that the coarse mesh in the stagnation region of the body generates a region of high entropy in these meshes $(1,2 \& 4)$. The entropy layer convects downstream and weakens the passage shock on the A4. Figure 4 shows the axisymmetric projection of mesh 4 , while figure 5 shows the corresponding entropy layer. Pressure contours plotted from the axisymmetric flow show that contour lines are connecting to the inlet. This indicates that the upstream boundary is too close. In mesh 5 the inlet was moved upstream with the same mesh packing (coarse) near the centerbody stagnation region. There was almost no change in either the integrated quantities or the entropy generation.

As shown in figure 3 , the front row is converging well with $\mathrm{N}=40$. The aft row shows a change in trend with increasing $N$, which implies that the aft row would require more than $N=40$. Examination of the 3-D flowfields showed that the change in trend is due to a sharpening of the passage shock in the root region of the $A 4$. The current version of this code requires that the mesh have a common axisymmetric projection for all axial-radial mesh surfaces. Since the position of passage shock on the A4 varies axially in the blade passage, to get good resolution of this shock with these meshes requires a fine mesh over a substantial portion of the blade chord. Hence, for the A4, more than $\mathrm{N}=40$ is required.

To correct for these mesh problems two additional meshes were used. Mesh 6 extends farther upstream and has more packing in the stagnation region. The increased mesh packing reduces the entropy generation and weakens the entropy layer, which results in a stronger passage shock. The final mesh improvement involves packing the mesh at the location of the blade passage shocks as indicated by the axisymmetric flowfield. Mesh 7 , with $\mathrm{N}=40$, produces essentially the same result as mesh 6 and is the model for all meshes used in the subsequent studies. There is still a change in $C_{p}$ between $N=20$ and $N=40$, but it is improved in this mesh. Figure 6 shows the axisymmetric projections of mesh 7 to illustrate a good $\mathrm{N}=40$ mesh and figure 7 shows the corresponding entropy layer.

All calculations reported here were run on a Cray Y-MP. Run times for the $\mathrm{N}=40$ meshes are rough 1 y $2.53 \times 10^{-5}$ second/cycle/point. Memory requirements are roughly $3.25 \times 10^{-5}$ Megaword/point. The smaller runs achieve convergence of integrated performance quantities when the average $\mathrm{d}(\rho) / \mathrm{dt}$ residual has dropped 2 to 2.5 orders of magnitude. The largest meshes required at least 
three passes (one pass consists of a front row and an aft row solution) of 800 time steps to obtain a convergence of 2.5 to 3 orders of magnitude.

The grid density study used the blade setting angles set in the wind tunnel test. The $C_{p}$ values for the experiment, listed in table II, are shown in figure 3 with those for the mesh study. These data show that the predicted performance with the experimental blade angles does not agree with experiment. The Euler code overpredicts the front row power by $18 \%$ and underpredicts the aft row power by $13 \%$ at these angles.

To improve the comparisons a flowfield with $C_{p}$ matched to the experiment, mesh 13, is predicted in addition to the matched blade angle flowfield. Table IV lists the blade angles and power coefficients of each blade row for these two conditions. Note that to match the power coefficient, the front blade angle was reduced by 1.3 degrees, and the rear increased by 0.6 degrees. In the experiment, the power coefficient of each rotor is measured by a balance located within the model nacelle. The thrust and power coefficient from the Euler predictions is calculated by integrating the surface static pressure on the blade. Ignoring viscous effects should change the integrated quantities by only a few percent, so the differences here are not felt to be completely a viscous effect.

Once generated, the predicted flowfield velocities are interpolated along mesh lines to the axial locations of the LDV data. The predictions are then processed through the LDV data plotting software. Figure 8 shows the predicted velocity components at axial station 5 . These components, at a fractional radius of $r / R=1.0$, are for meshes 7,8 , and $9(40,20$ and 10 points per chord respectively). They show the effect of mesh density on velocity details. The $N=10$ mesh differs significantly from the $N=20$ mesh, but the differences between $\mathrm{N}=20$ and $\mathrm{N}=40$ are smaller. The convergence in the velocity components echoes the convergence seen in the integrated quantities but will require a greater mesh density.

Extrapolating from the $N=10,20$, and 40 point meshes, an $N=80$ point mesh would require roughly 4 million grid points and have a run time of 50 hours. Runs of this size are currently impractical, so this paper will examine the accuracy of $\mathrm{N}=40$ point mesh predictions.

\section{Comparisons with LDV Data}

The first code validation is a comparison of predicted and measured circumferentially averaged tangential velocities. The comparison, shown in figure 9 , is for $r / R=0.8$ at axial stations $2,3,4$, and 5 . The LDV data curve shows lower tangential velocities than either of the Euler predictions (matched power and matched blade angle) and indicates that the magnitude of the tangential velocity increases significantly with increased distance downstream of the front rotor. This implies that the swirl and the angular momentum of the flow are increasing downstream of the rotor. This is not correct since to increase the angular momentum work must be done on the flow. Downstream of the rotor the axial variation of the tangential velocity at a given radial location should be relatively constant, with only slight changes occurring due to slipstream contraction. Although they differ in level, the shapes of the curves corresponding to the Euler solutions reflect this behavior. 
The LDV data curve of figure 9 indicates a particle lag problem. That is, the LDV seed particles (atomized droplets of dioctyl phthalate) appear to be too big to follow the flow. Calculations indicate that particles with diameters between one and two microns would be small enough to follow the shock-free flow outside the blade passages. A much larger particle, on the order of six microns in diameter, would respond in a manner similar to the solid curve in figure 9 . This curve was generated using the technique of reference 11, with the particle response fitted to the LDV data. The curve corresponds to a six micron particle subjected to a 67 foot/second step change in tangential velocity at roughly the $3 / 4$ chord position of the front rotor blades. This predicted response is nearly identical to the data curve.

The particle lag errors illustrated by the above comparison at $r / R=0.8$ represent a "worst case" example because this is the innermost location at which axial and tangential velocities were measured. As pointed out in reference 9 , the mean particle size detected by the LDV system is expected to vary with measurement location. While positioned to acquire data in the horizontal plane, the laser beams reflect off the nacelle surface. As the probe volume is zoomed in towards the nacelle, the LDV system's receiving optics detect a larger portion of the light reflected off the body. This reflected light appears as electronic noise on the signals created by the particles passing through the probe volume. As the noise level increases, the weaker signals become buried in the noise, and it is increasingly more difficult to measure the signal frequencies and, therefore, the particle velocities. On the average, the weaker signals are produced by the smaller particles passing through the probe volume. Therefore, closer to the nacelle the average size of the particles detected by the LDV system increases. The mean particle size further out near the tip should be somewhat less than six microns. The radial velocities, which were measured in a vertical plane above the model, should be relatively free from particle lag bias errors.

Figure 9 indicates that the differences due to particle lag between the data and the Euler solutions decrease with distance downstream of the rotor. Therefore, it is best to make comparisons at an axial location relatively far downstream. The data obtained at axial station five will be used in the comparisons that follow.

It should be pointed out that in the following comparisons the data of reference 9 has been shifted circumferentially so that the locations of the blade wakes more closely match those predicted by the Euler analysis. Originally (for reference 9), the circumferential placement of the data relative to the blades was determined based on data obtained during intrablade surveys. False velocity measurements due to reflections off the biades ("blade flash") were used to estimate the locations of the blades in the data. This only provides an approximation of the blade location, however, due to uncertainty as to the exact part of the blade generating the blade flash. Initial comparisons between the data and analysis indicated a difference in the wake locations of approximately 2.8 degrees. Since it is not known whether these differences are real or the result of an inaccurate estimate of the blade location, the experimental data was shifted to provide a better match with analysis.

Figures 10 and 11 present the $F 4$ axial velocity flowfield for the entire station 5 plotted from the LDV data and mesh 7 Euler prediction. The 
crossflow velocities are presented in figures $12 \& 13$. Station 5 is just ahead of the leading edge of the A4 blade row. Only the radial locations at which the LDV measured all three velocity components are shown. (Further inboard the laser beam reflections off the nacelle surface prevented measurement of the axial and tangential components.) The view depicted in figures 10 through 13 is from downstream of the front rotor, looking upstream. The outlines of three front rotor blades and the hub contour show the relative locations between the velocity measurements, predictions, and the propeller blades. In this view, propeller rotation is counter-clockwise.

The gray scale contours of axial velocity in figures 10 and 11 show that the prediction is fairly similar to the measured data. The major differences are that the Euler prediction has higher velocities inboard and lower velocities outboard than are measured. Numerical dissipation is evident in the larger size of the tip vortex and the diffused blade wake. Since the LDV data is the average over a large number of rotor revolutions, any variation of vortex position would diffuse the measured vortex. Therefore, the actual vortex core diameter would be no larger than indicated by the LDV data.

The Euler code prediction also suggests a larger region of reduced axial velocity outboard of the core of the tip vortex. This axial velocity defect results because the tip vortex is convected downstream along a helix at an angle to the axial direction. In regions outboard of the core the velocity field of the tip vortex has a component in the upstream direction. The larger size of the axial defect region indicates that the Euler code is overpredicting the tip vortex strength.

The crossflow velocity vectors in figures 12 and 13 show that the location of the tip vortex is accurately predicted. Here again, it is evident that the Euler code overpredicts both the strength and core diameter of the tip vortex. Since the blade geometry used is only the predicted hot shape, there may be some tip deflection that can account for a higher predicted tip loading and therefore a stronger tip vortex. The LDV data shows a sharp change in velocity direction across the blade wake. The Euler solution has a diffuse wake and shows only a gradual change of velocity direction across it.

Generally, the secondary velocities predicted by the Euler code are higher than those measured with the LDV. These predicted secondary velocities may be higher due to a combination of the neglect of viscosity in the analysis, and the particle lag decreasing the velocities in the LDV data.

The larger vortex core in the Euler prediction is probably due to a lack of resolution in the large cells behind the rotor. Near the rotor the cells are very small and the smearing effect is smaller. The mesh stretches in the circumferential direction, and the mid-passage cell width is roughiy the size of the core diameter of the tip vortex. Since the mesh does not follow the blade wakes or the tip vortex, the small scale flow features dissipate in the large cells. Adapting the mesh in the circumferential direction to follow the wake would improve the tip vortex resolution downstream.

To see the details of the flow another way, figures 14,15 , and 16 show station 5 velocity components at three radial locations. Figure 14 corresponds to $r / R=1.0$, just outboard of the tip vortex core. All three velocity components show that the predicted tip vortex perturbation is wider than in the LDV data. The axial velocity perturbations are very similar in 
magnitude to the LDV data, while the tangential and radial components are overpredicted by the Euler code. The largest discrepancy is in predicted radial perturbation, which is twice as large as the experiment. The predicted radial velocity is in phase with the LDV data, but there is a slight phase shift in the axial and tangential components.

The data in figure 15 is at the location closest to the center of the tip vortex: $r / R=0.96$. At this station the predicted axial perturbation is nearly zero, indicating that in the Euler solution this is the center of the vortex. The magnitude of the predicted radial velocity perturbation is nearly correct, but shows that the predicted vortex has about twice the diameter.

The third radial cut, figure 16 , is at $r / R=0.86$. This is inboard of the tip vortex and mostly shows that the predicted radial perturbations are very good in the blade wake. It also shows that the LDV tangential velocities with particle lag are very different than the prediction.

A final comparison at station 5 is in figure 17 where the axisymmetric average of the flowfield is presented. This figure highlights the differences in axial and tangential velocity inboard of the blade tip. It also shows that the flow outboard of the tip is not being predicted we11. The presence of the LDV window would tend to increase the blockage, and therefore increase the axial velocity in the freestream flow. But, this only accounts for part of the difference.

\section{Conclusions}

Comparisons made here between Euler predictions and LDV measurements for velocities in the vicinity of an advanced counterrotating pusher propfan are in general good. These comparisons lead to the following conclusions:

1. A dense mesh near the stagnation region of the centerbody is needed to accurately predict the aft row passage shock strengths.

2. A minimum of 40 points on a chord line is required to accurately predict the integrated blade performance. For the F4 blade, which has no passage shock at the hub, $N=40$ is sufficient. For the A4 blade, which does have a passage shock, more than 40 points are needed to resolve the passage shock.

3. To get converged flow field details requires more points than converged integrated quantities. Comparisons of $N=10,20$ and 40 point meshes suggest that more than $N=40$ should be investigated.

4. The predicted tip vortex locations are very good though the vortex strength is high, and the core size is too large. The explanations are that neglecting viscosity increases the strength, and the numerical dissipation in large cells behind the blade expands the core diameter. An improvement would be to align the mesh with the flow to keep the tip vortex in the tight mesh. 


\section{REFERENCES}

1. Adamczyk, J.J.: Model Equation for Simulating Flows in Multistage Turbomachinery. ASME Paper 85-GT-226, Nov. 1984. (NASA TM-86869)

2. Adamczyk, J.J.; Mulac, R.A.; and Celestina, M.L.: A Model for Closing the Inviscid Form of the Average-Passage Equation System. ASME Paper 86-GT-227, June 1986. (NASA TM-87199)

3. Bober, L.J.; Chaussee, D.S.; and Kutler, P.: Prediction of High Speed Propeller Flow Fields Using a Three-Dimensional Euler Analysis. AIAA Paper 83-0188, 1983 (NASA TM-83065).

4. Denton, J.D.: Time Marching Methods for Turbomachinery Flow Calculations. Numerical Methods in Applied Fluid Dynamics, B. Hunt, ed., Academic Press, New York, 1980, pp. 473-493.

5. Hanson, D.B.: Near-Field Frequency Domain Theory for Propeller Noise. AIAA Journal, Vol. 23, 1985, pp499-504.

6. Janus, J.M.; and Whitfield, D.L.: A Simple Time Accurate Turbomachinery Algorithm with Numerical Solutions of Uneven Blade Count Configuration. AIAA-88-0206, January 1989.

7. Swallow, R.J.; and Aiello, R.A.: NASA Lewis 8- by 6-Foot Supersonic Wind Tunne1. NASA TM X-71542, 1974.

8. Hughes, C.E.; and Gazzaniga, J.A.: Summary of Low-Speed Wind Tunnel Results of Several High-Speed Counterrotation Propeller Configurations. AIAA paper 88-3149, JuTy 1988. (NASA TM-100945)

9. Podboy, G.G.; and Krupar, M.J.: Laser Velocimeter Measurements of the Flowfield Generated by an Advanced Counterrotating Propeller. AIAA Paper 89-0434, January 1989. (NASA TM-101437)

10. Celestina, M.L.; Mulac, R.A.; and Adamczyk, J.J.: A Numerical Simulation of the Inviscid Flow Through A Counter-Rotating Propeller. Journal of Turbomachinery, Vo1. 108, No. 4, October 1986, pp. 187-193.

11. Feller, W.V.; and Meyers, J.F.: Development of a Controllable Particle Generator for LV Seeding in Hypersonic Wind Tunnels. Minnesota Symposium on Laser Anemometry, October 1975, pp. 345-357. 
Table I. - Design characteristics of the F4/A4 counterrotating propeller model.

$\begin{array}{lr} & \\ \text { Design Mach number } & 0.72 \\ \text { Advance ratio, J } & 2.80 \\ \text { Design total power coefficient, PQA } & 4.15 \\ \text { Design total thrust coefficient, TQA } & 1.26 \\ \text { Disk loading, shp/A } & 86 \\ \text { Number of blades } & 8 / 8 \\ \text { Total activity factor } & 2456 \\ \text { Tip speed (rotor 1), ft/sec } & 787 \\ \text { Aerodynamic tip sweep, deg } & 19 / 20 \\ \text { Inlet radius ratio } & 0.424 \\ \end{array}$

Table II. - LDV test conditions for the F4/A4 propeller model.

\begin{tabular}{llllll}
\hline & Minimum & Maximum & Mean & \multicolumn{2}{l}{ Standard Deviation } \\
READING & 2860 & 2880 & & & \\
Mach & 0.7080 & 0.7121 & 0.7100 & 0.0011 & $(0.15 \%)$ \\
$\mathrm{J}_{1}$ & 2.7676 & 2.7899 & 2.7786 & 0.0053 & $(0.19 \%)$ \\
$\mathrm{J}_{2}$ & 2.7584 & 2.7798 & 2.7683 & 0.0051 & $(0.18 \%)$ \\
$\mathrm{C}_{p_{1}}$ & 1.3887 & 1.4153 & 1.4019 & 0.0083 & $(0.59 \%)$ \\
$\mathrm{C}_{p_{2}}$ & 1.2656 & 1.3162 & 1.2980 & 0.0113 & $(0.87 \%)$ \\
$\mathrm{t}_{\mathrm{t}}$ & 542.17 & 547.37 & 544.08 & 1.53 & $(0.28 \%)$ \\
$\mathrm{t}_{\mathrm{s}}$ & 492.57 & 497.42 & 494.25 & 1.42 & $(0.29 \%)$ \\
$\mathrm{P}_{\mathrm{t}}$ & 2490.74 & 2511.88 & 2498.77 & 7.17 & $(0.29 \%)$ \\
$\mathrm{P}_{\mathrm{s}}$ & 1779.83 & 1796.33 & 1785.31 & 5.52 & $(0.31 \%)$ \\
& & & & & \\
\hline
\end{tabular}

Table III. - Grid density study mesh dimensions.

\begin{tabular}{llllllllll}
\hline \multicolumn{1}{l}{} \\
\multicolumn{1}{l}{ Details of } & \multicolumn{1}{c}{ the } & grids & \multicolumn{1}{c}{ used in the density study are: } \\
Mesh & IL & JL & KL & ILE1 & ITE1 & ILE2 & ITE2 & JTIP \\
1 & 52 & 16 & 11 & 13 & 23 & 32 & 42 & 9 \\
2 & 103 & 31 & 21 & 25 & 45 & 63 & 83 & 17 \\
4,5 & 205 & 61 & 41 & 49 & 89 & 125 & 165 & 33 \\
6,7 & 205 & 61 & 41 & 65 & 105 & 139 & 179 & 33 \\
8 & 103 & 31 & 21 & 33 & 53 & 70 & 90 & 17 \\
9 & 52 & 16 & 11 & 17 & 27 & 35 & 45 & 9 \\
13 & 205 & 61 & 41 & 65 & 105 & 139 & 179 & 33 \\
\end{tabular}


Table IV. - Match $C_{p}$ and matched $\beta$ conditions.
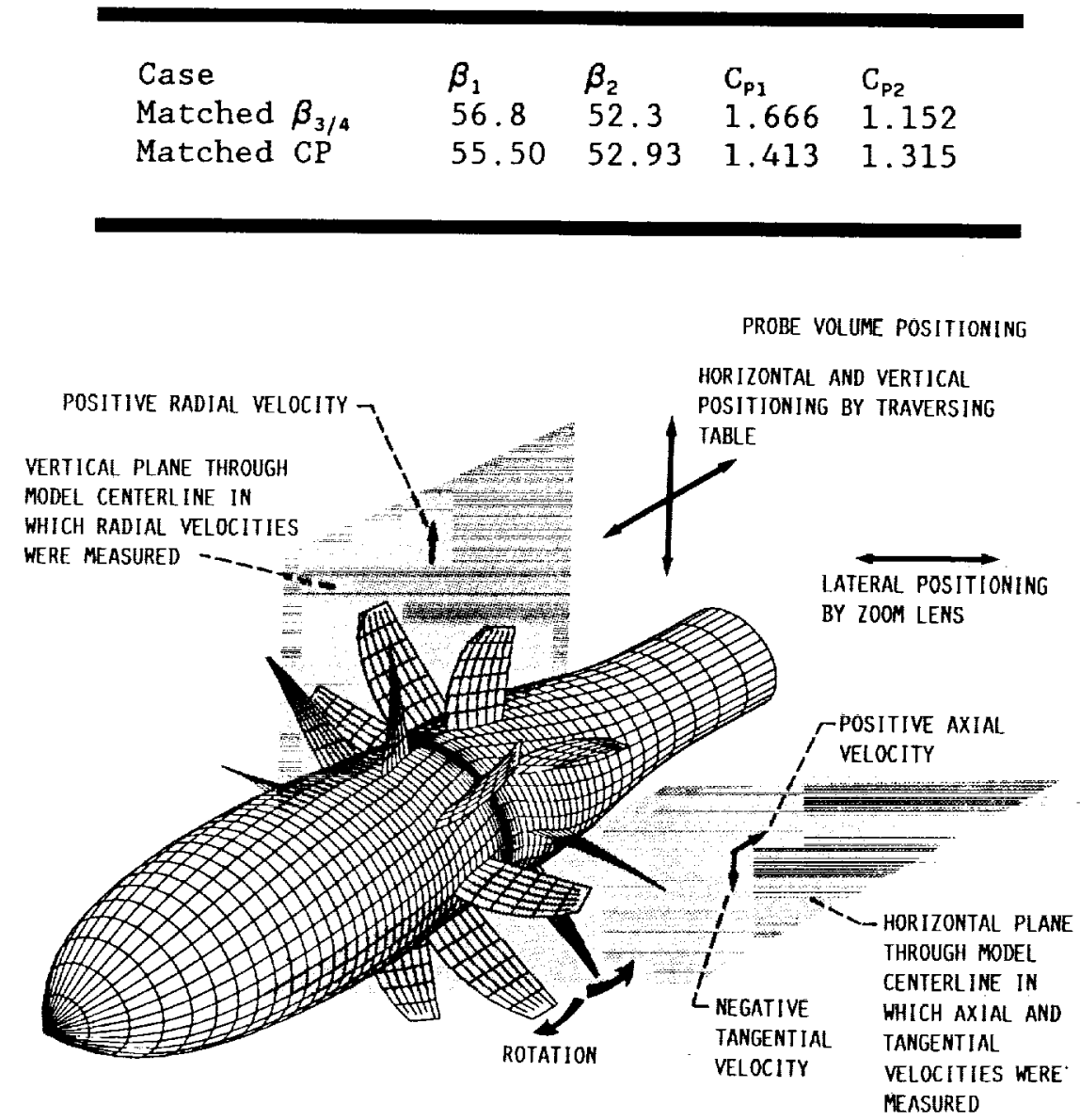

Figure 1. - Schematic of propeller model illustrating LDV probe volume positioning and horizontal and vertical measurement planes.

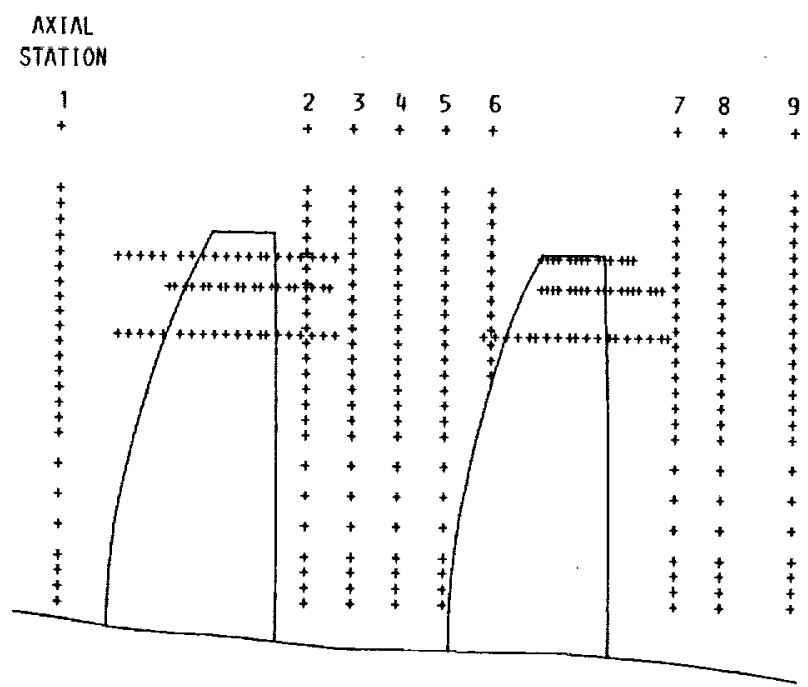

Figure 2. - Meridional view of propeller model showing LDV measurement locations.

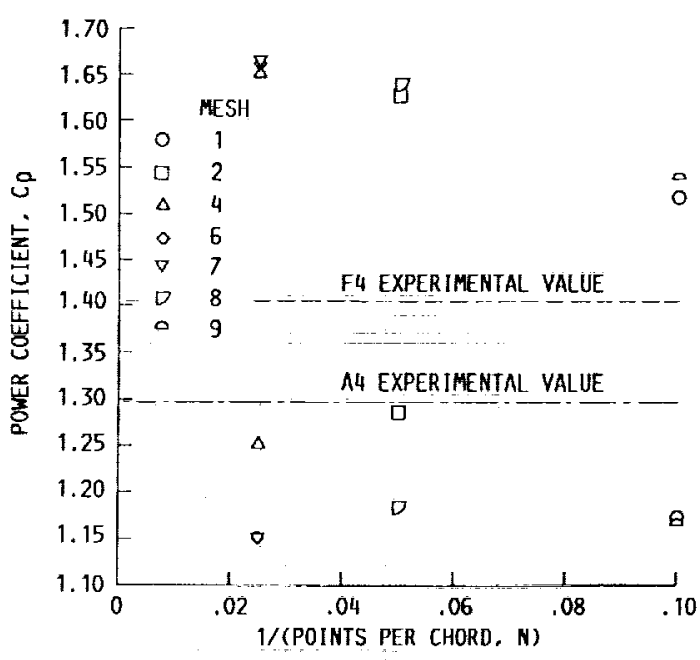

Figure 3. - Sensitivity of power coefficient to grid density. 


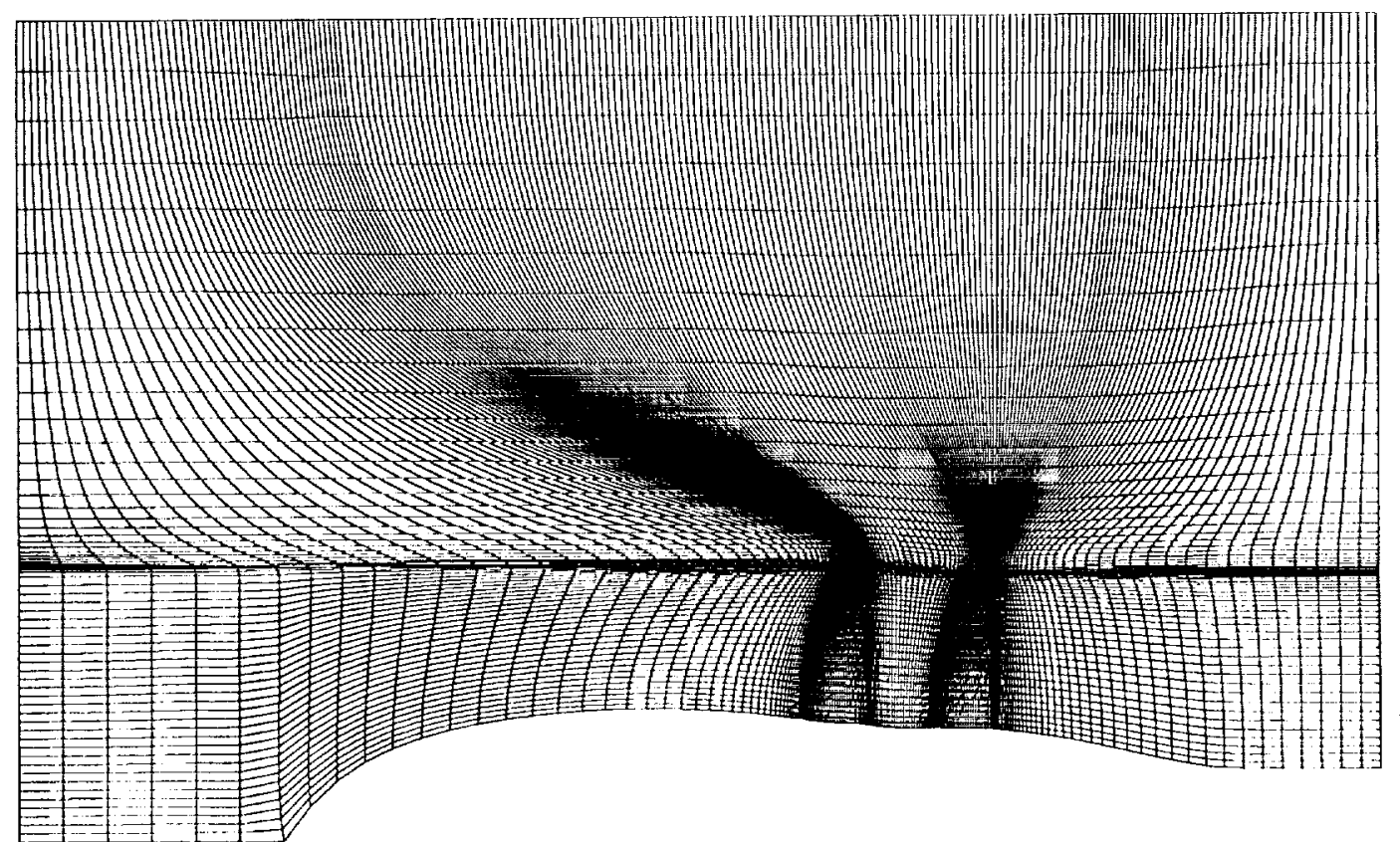

Figure 4. - Axisymmetric projection of mesh 4.

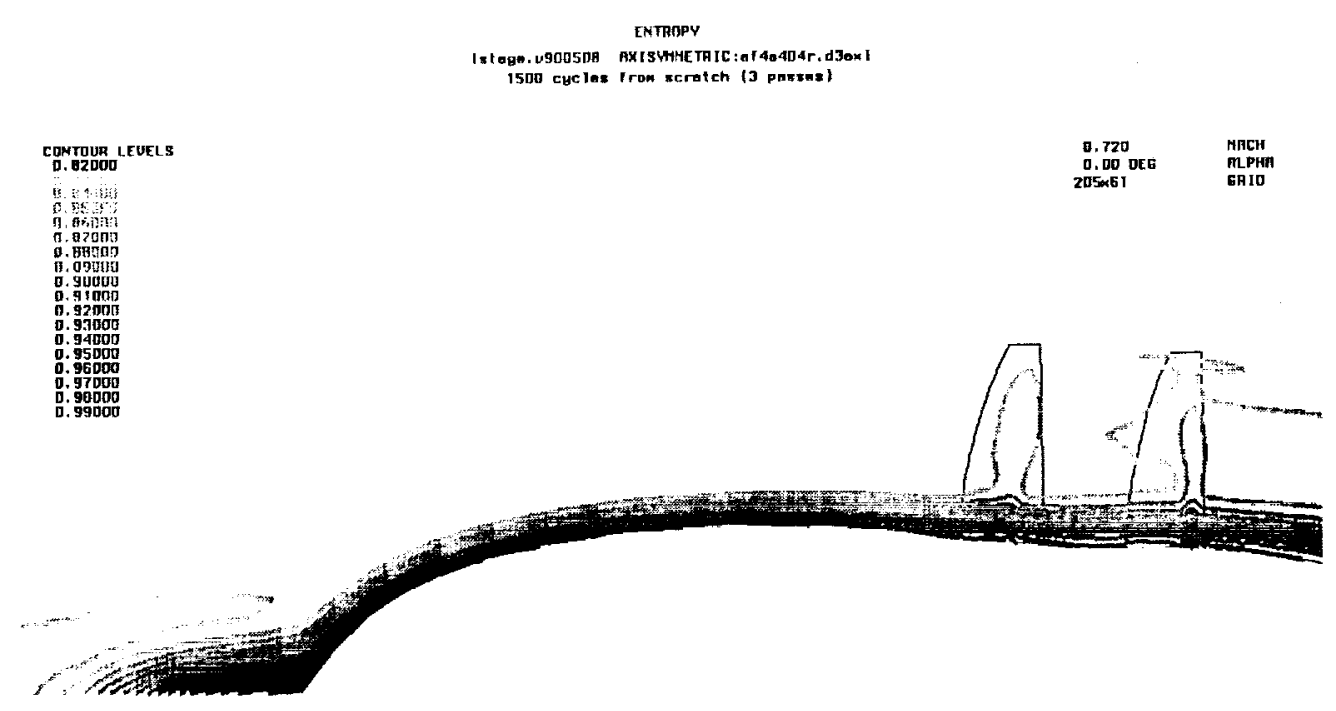

Figure 5. - Entropy layer generated in mesh 6 stagnation region. 


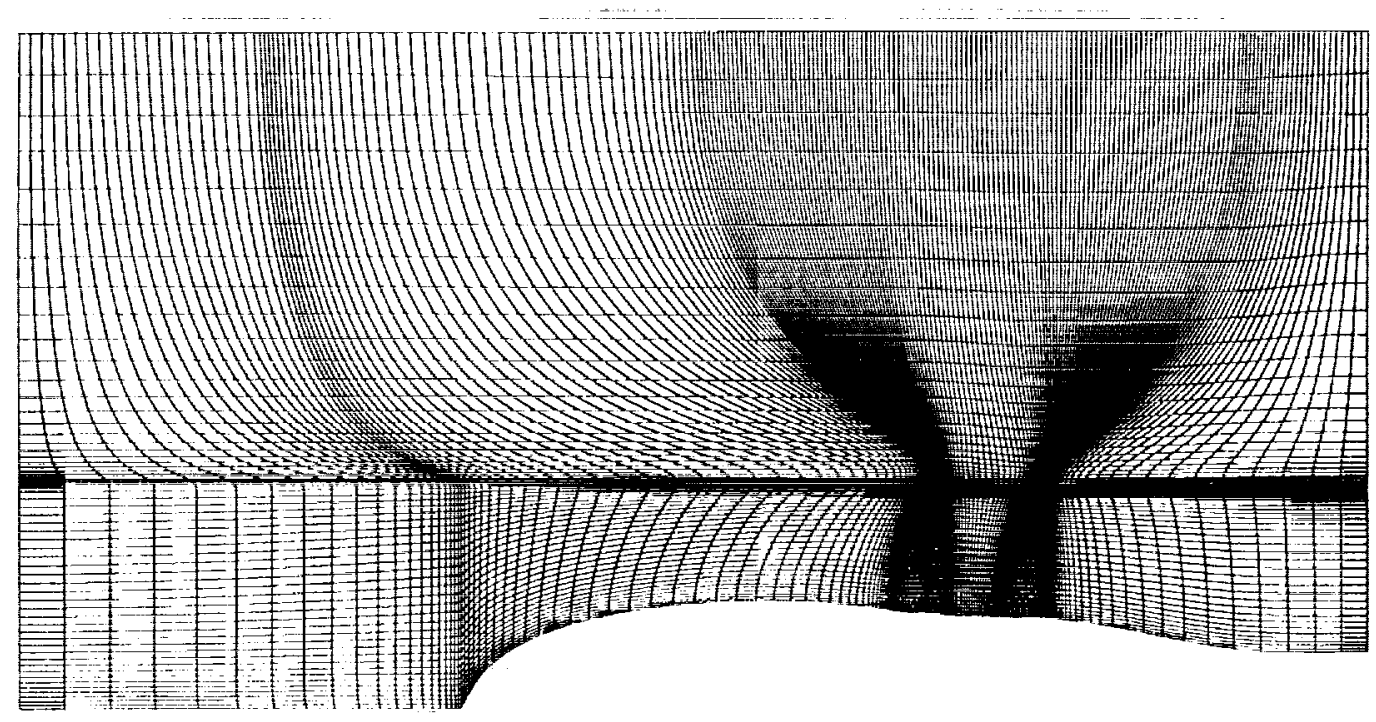

Figure 6. - Axisymmetric projection of mesh 7 .

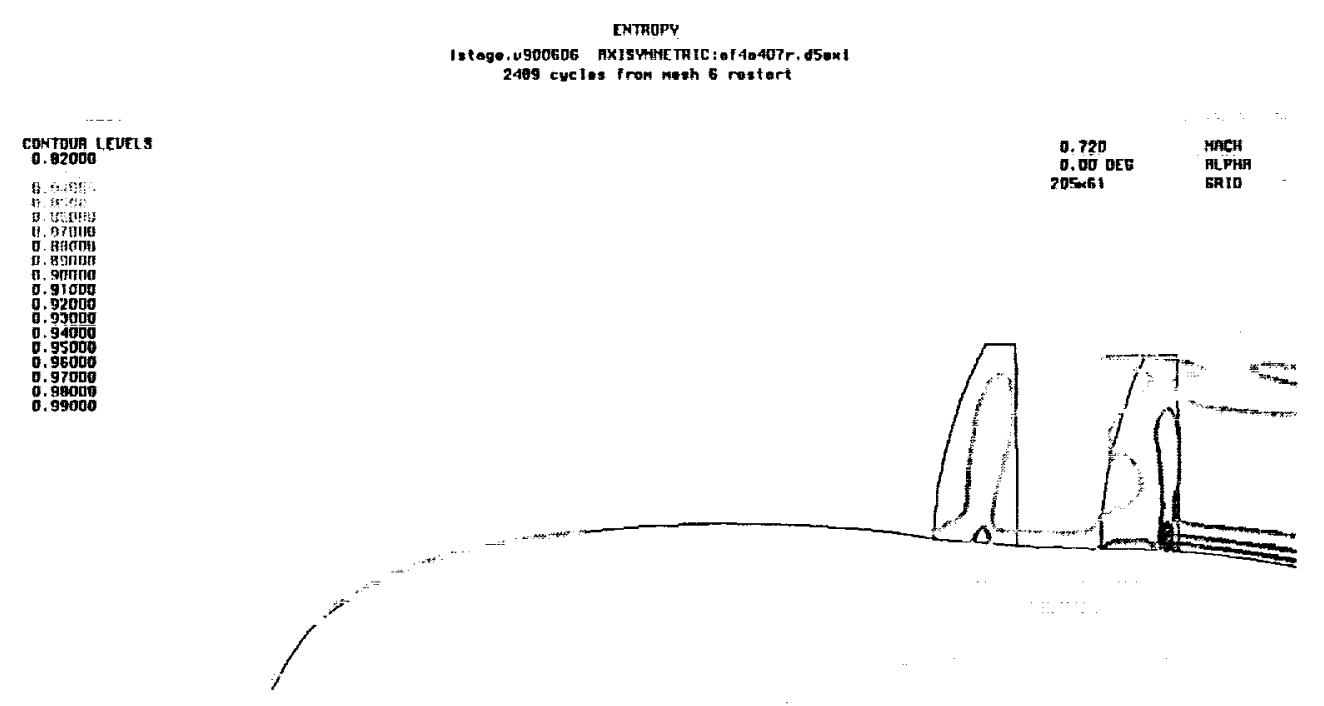

Figure 7. - Entropy layer generated in mesh 7 stagnation region. 

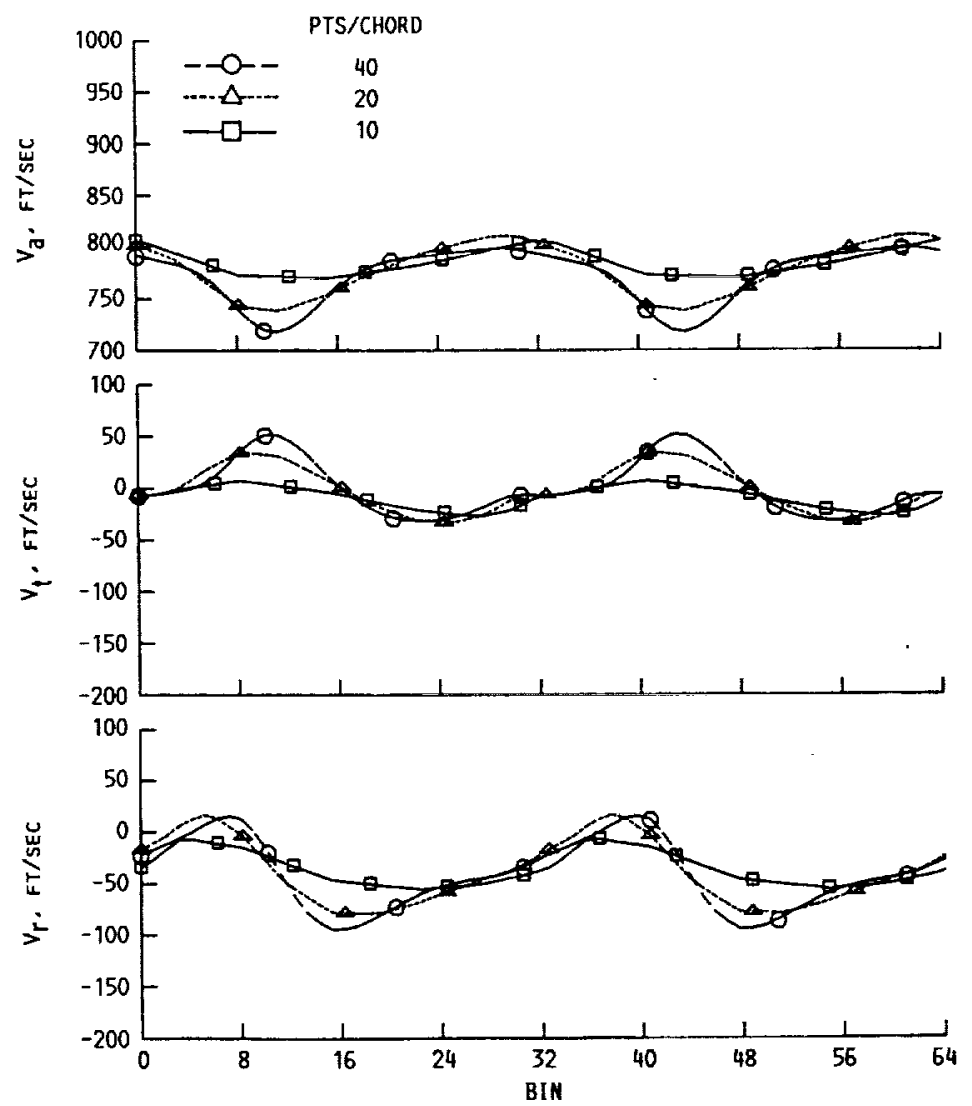

(a) Axial components.

(b) Tangential components.

(c) Radial components.

Figure 8. - Mesh density effect on predicted velocity details at $r / R=1.0$.

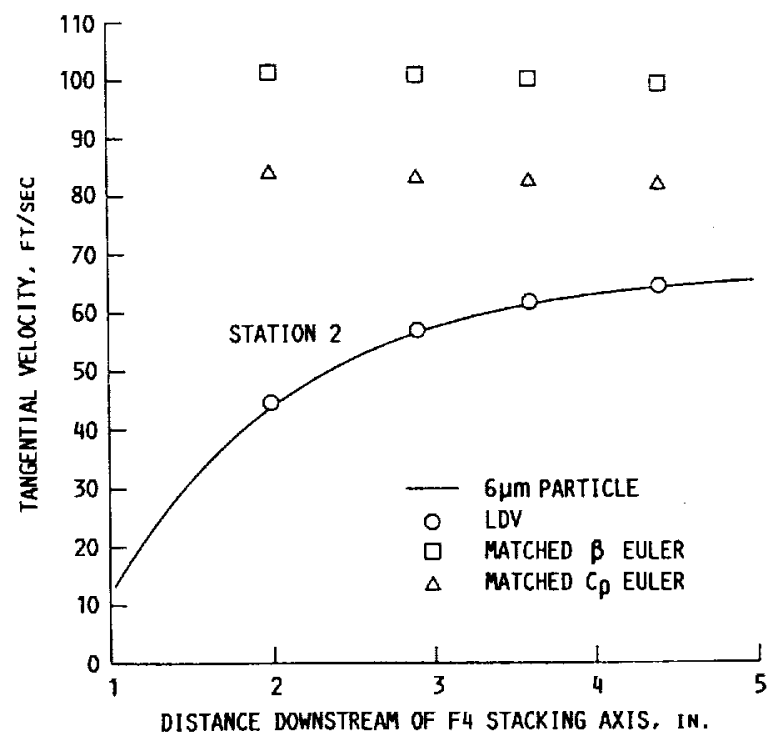

Figure 9. - Axial variation of tangential velocity at $r / R=0.8$. 


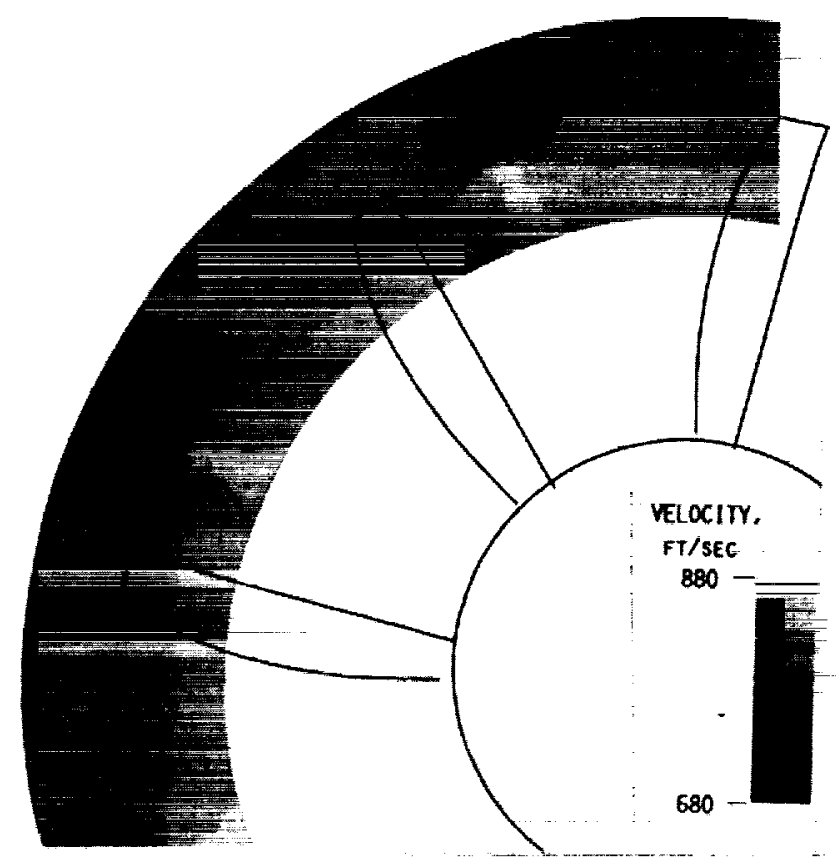

Figure 10. - LDV measured axial velocities at station 5.

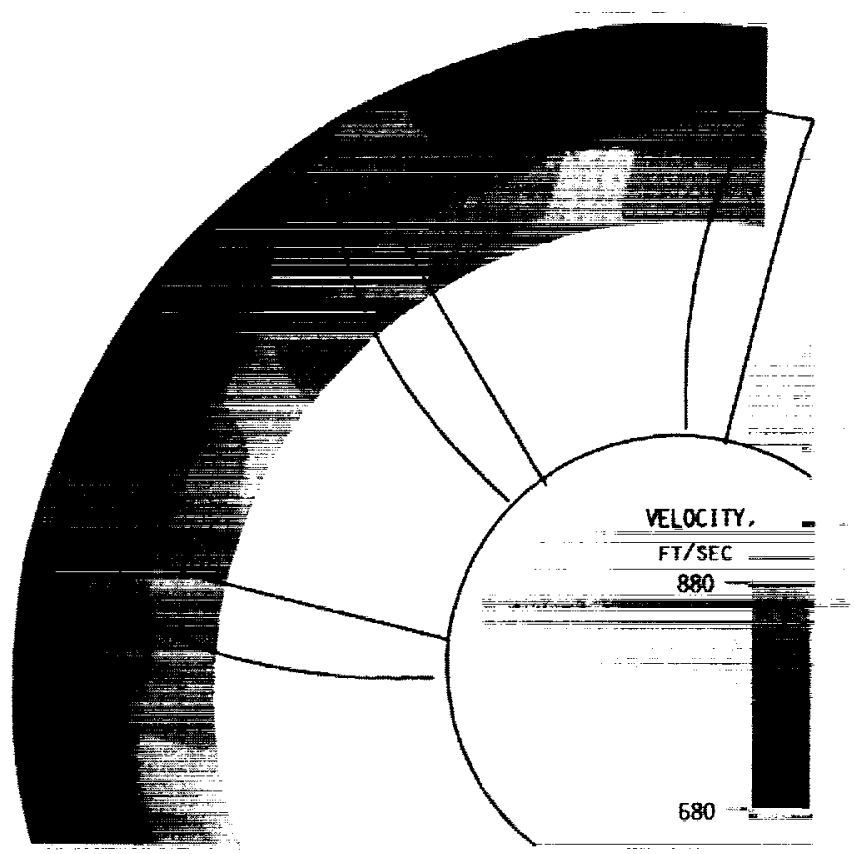

Figure 11. - Euler predicted axial velocities at station 5. 


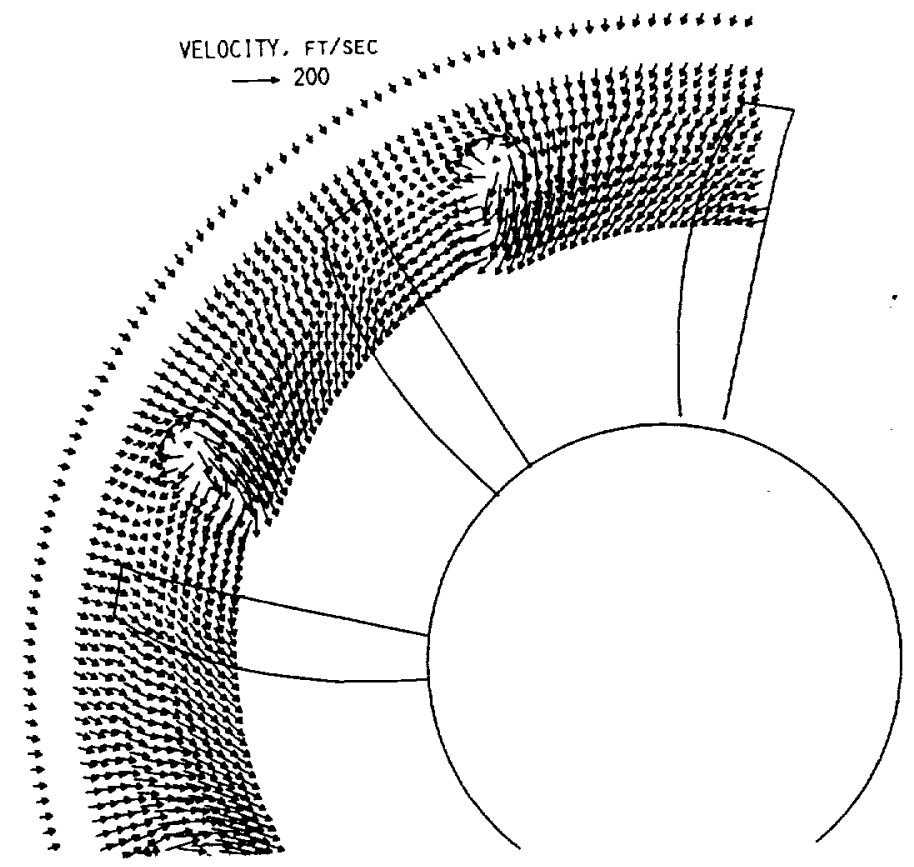

Figure 12. - LDV measured crossflow velocity field at station 5.

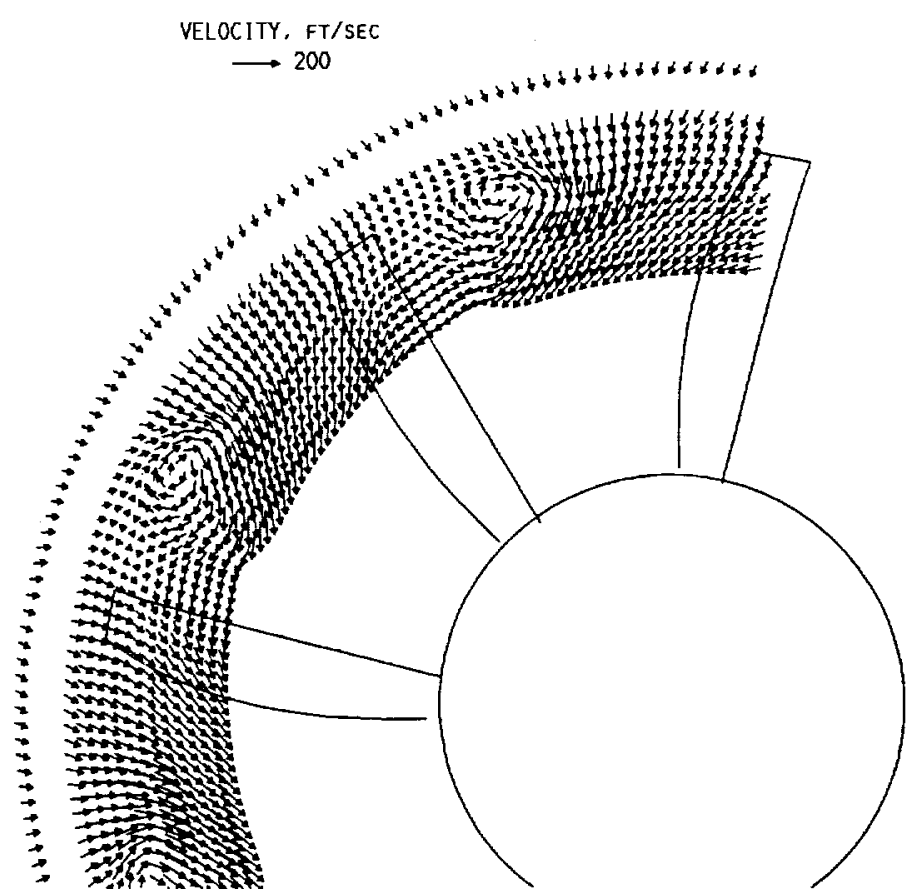

Figure 13. - Euler predicted crossflow velocity field at station 5. 

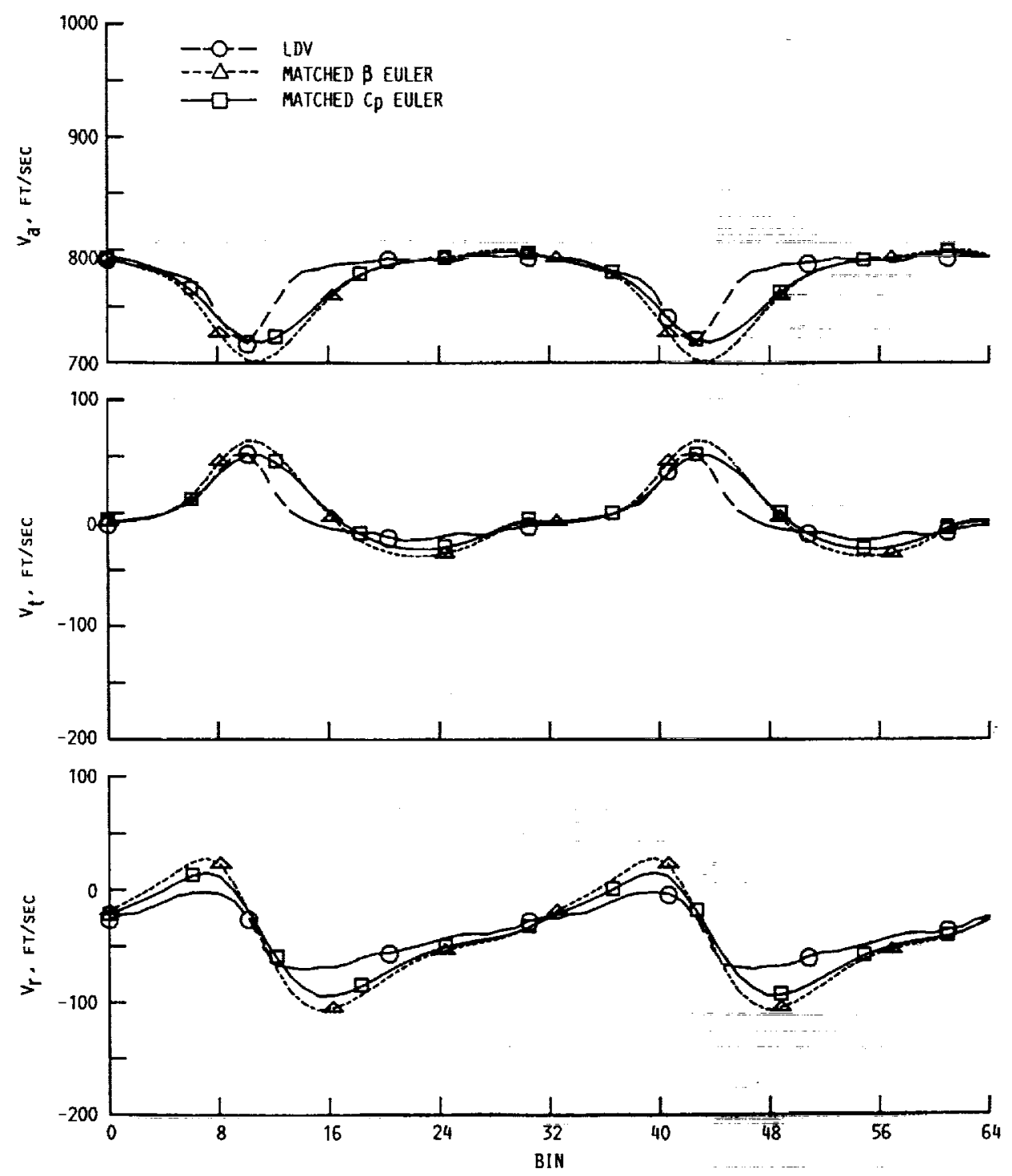

(a) Axial components.

(b) Tangential components.

(c) Radial components.

Figure 14. - IDV measured and Euler predicted flowfield at axial station 5 for $r / R=1.0$. 

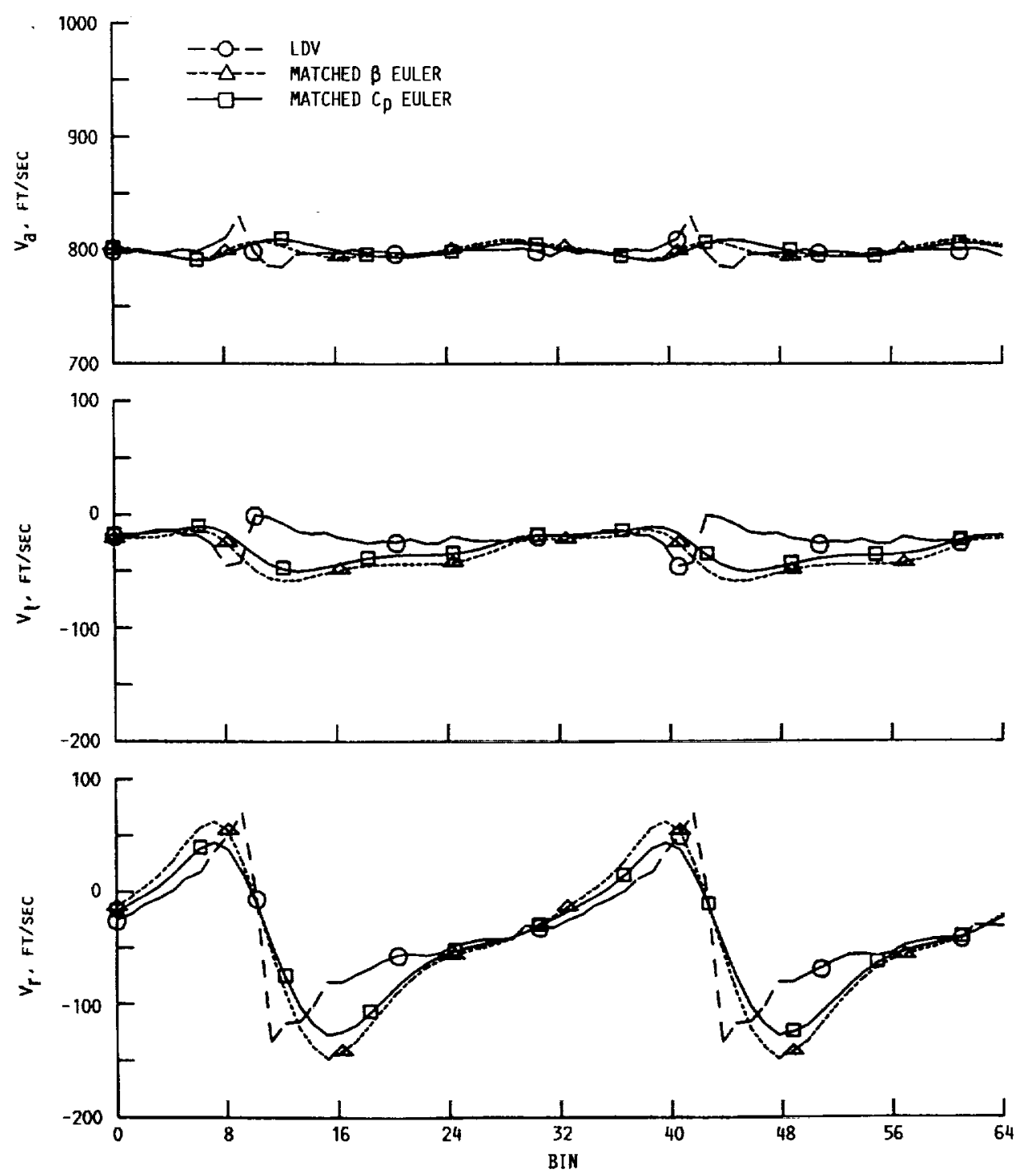

(a) Axial components.

(b) Tangential components.

(c) Radial components.

Figure 15. - LDV measured and Euler predicted flowfield at axial station 5 for $r / R=0.96$. 

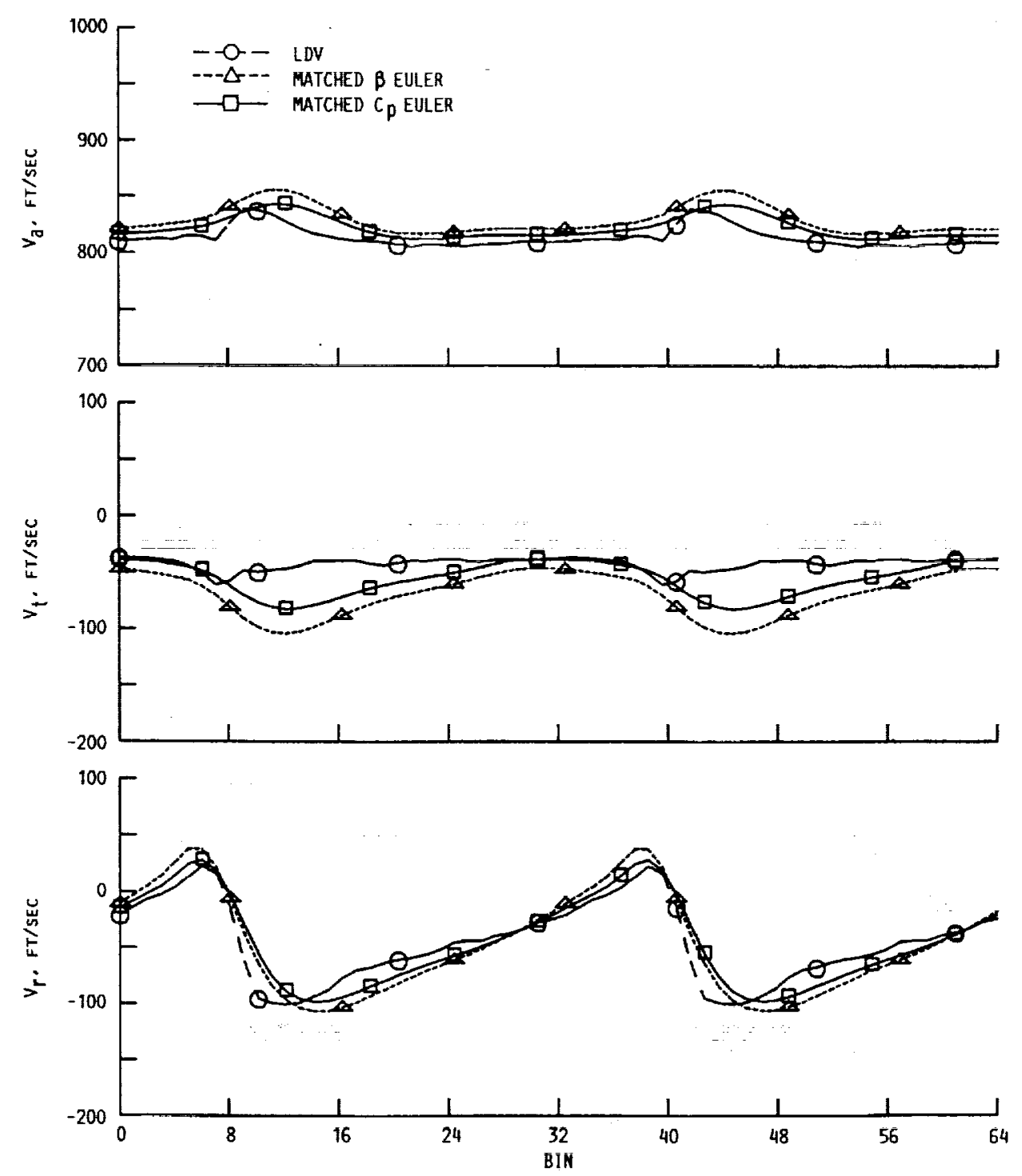

(a) Axial components.

(b) Tangential components.

(c) Radial components.

Figure 16. - LDV measured and Euler predicted flowfield at axial station 5 for $r / R=0.89$. 

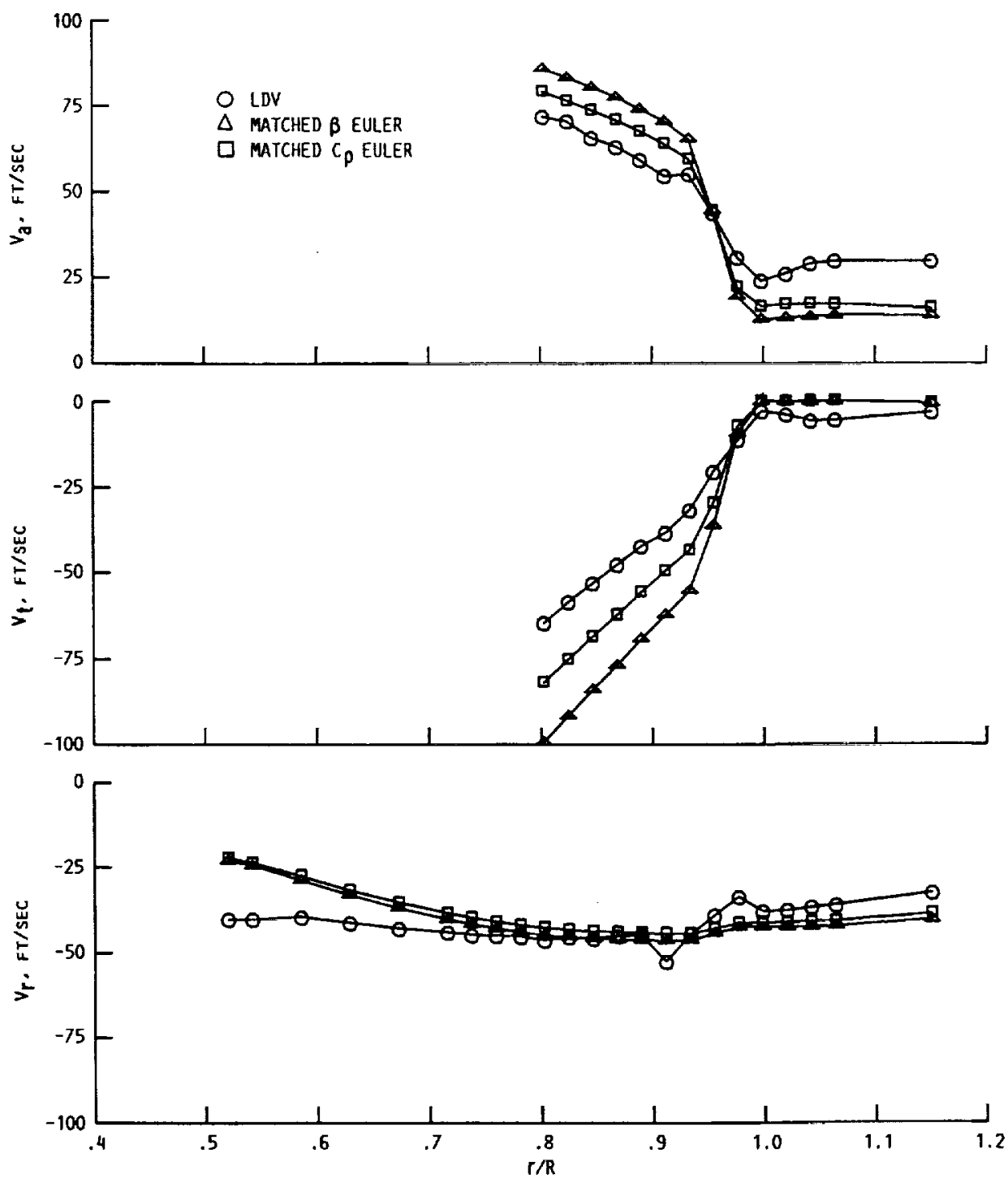

(a) Axial components.

(b) Tangential components.

(c) Radial components.

Figure 17. - Axisymmetric average radial distribution of LDV measured and Euler predicted velocities at station 5 . 


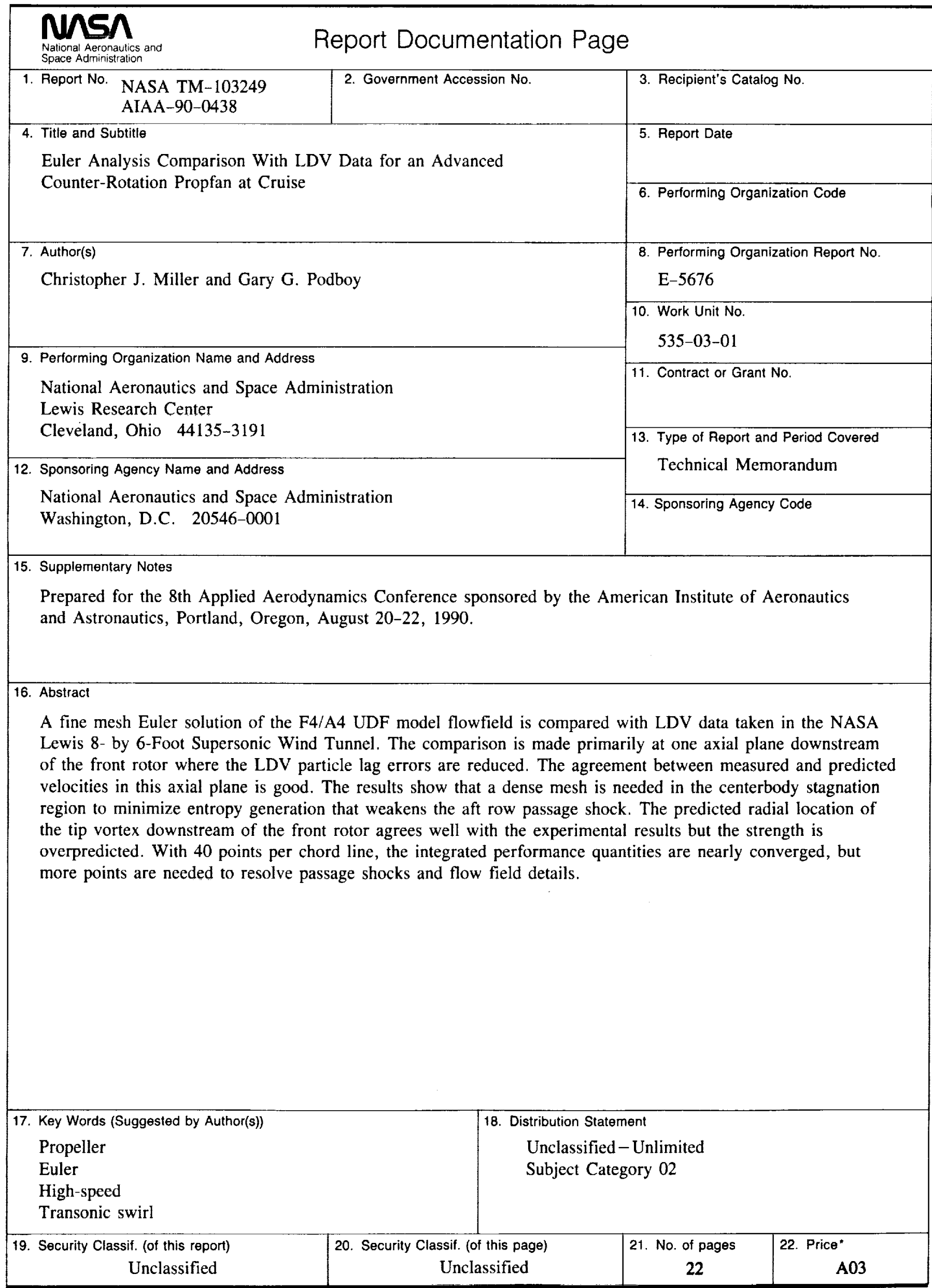

